\title{
Water Priorities for the Nation-The U.S. Geological Survey Next Generation Water Observing System
}

The challenges of providing safe and sustainable water supplies for human and ecological uses and protecting lives and property during water emergencies are well recognized. The U.S. Geological Survey (USGS) plays an essential role in meeting these challenges through its observational networks and renowned water science and research activities (National Academies of Science, Engineering, and Medicine, 2018). Substantial advances in water science, together with emerging breakthroughs in technical and computational capabilities, have led the USGS to develop a Next Generation Water Observing System (NGWOS). The NGWOS will provide real-time data on water quantity and quality in more affordable and rapid ways than previously possible, and in more locations. The data will be served through a modernized USGS National Water Information System that will be coupled to advanced modeling tools to inform daily water operations, decision-making during water emergencies (like floods, droughts, and contaminant spills), assessments of past trends in water quantity and quality, and forecasts of future water availability.

\section{NGWOS Design Strategy}

The USGS has a nearly 140 -year history of providing reliable and relevant scientific information to decision makers. Today (2019), the USGS operates and maintains real-time, continuous monitoring networks nationwide consisting of more than 8,200 streamflow-gaging stations, 2,100 water-quality stations, 1,700 groundwater-level monitoring wells, and 1,000 precipitation stations. USGS hydrographers make tens of thousands of discrete water measurements each year. Requests for USGS data exceed 670 million annually. Yet, the current National Streamflow Network - although providing data at critical locations - covers less than 1 percent of the Nation's streams and rivers. This sampling density helps to inform current and past water conditions (see, for example, WaterWatch) but is not sufficient for predicting interactions between climate, surface water, groundwater and soil moisture across large watersheds.

It is not necessary or feasible to collect data at a high spatial density throughout all large watersheds and aquifers. A more practical approach is to develop intensive monitoring networks in a small number of medium-sized watersheds $(10,000-20,000$ square miles) and underlying aquifers that are representative of larger regions across the Nation. Data from these intensively monitored watersheds can then be used, alongside data from existing monitoring networks, to construct and reduce the uncertainty in advanced models to fill in data and knowledge gaps in regional and national water assessments and predictions. At present, it is anticipated that the NGWOS will include at least 10 intensively monitored medium-sized watersheds, selected with input from USGS stakeholders, to represent a wide range of environmental, hydrologic, and landscape settings across the Nation.

NGWOS monitoring networks will integrate fixed and mobile monitoring assets in the water, ground, and air, including innovative webcams and new ground- and space-based sensors. When fully implemented, the NGWOS will provide high temporal- and spatial-resolution data on (1) streamflow; (2) water-cycle components, including evapotranspiration, snowpack, and soil moisture; (3) a broad suite of water-quality constituents, including temperature, nutrients, salinity, and turbidity; (4) connections

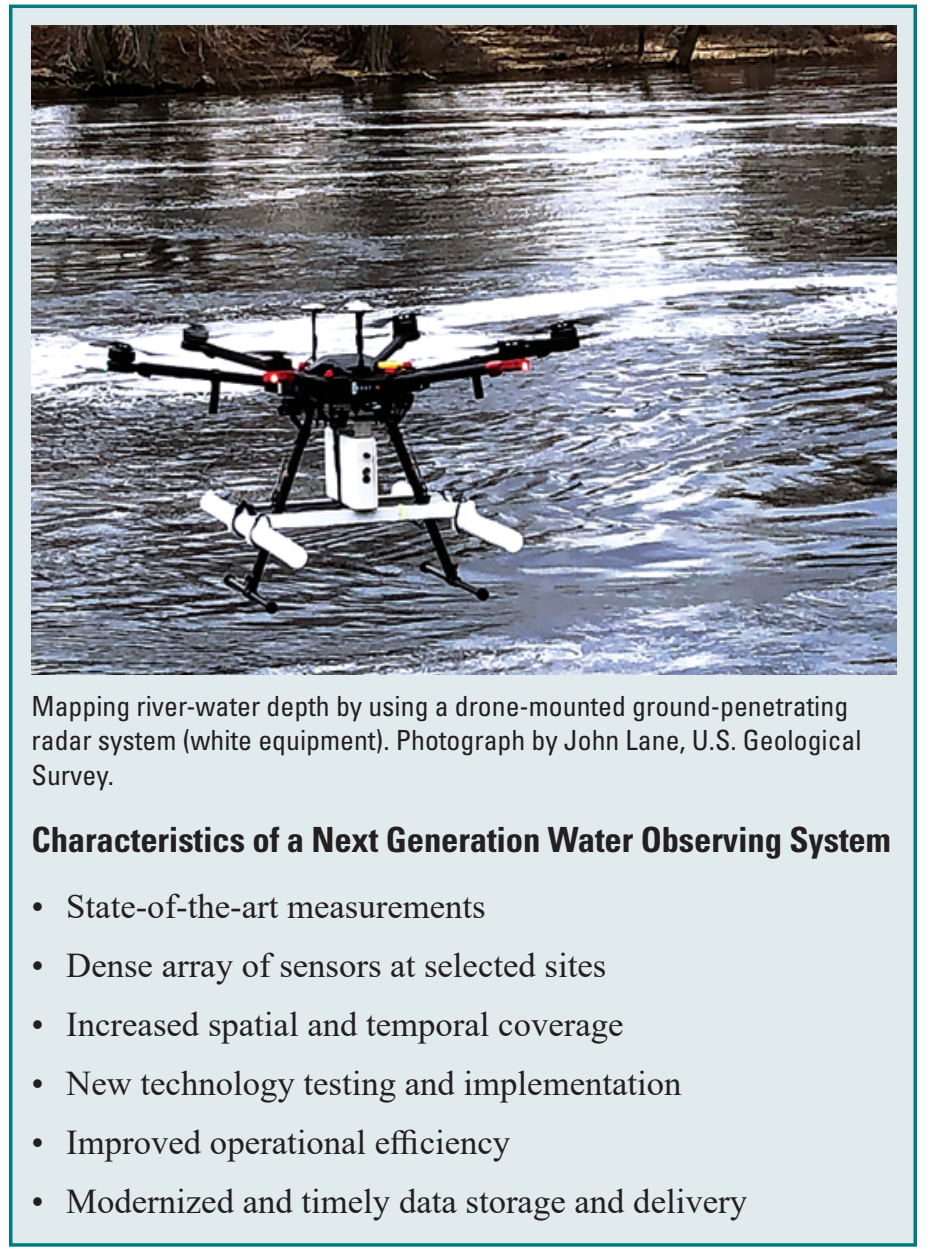

between groundwater and surface water; (5) stream velocity distribution; (6) sediment transport; and (7) water use.

The USGS information systems for water-data management and delivery are being transformed and modernized as part of the NGWOS to accommodate new data and sensor networks, allow for integration with water data from multiple agencies and sectors, display observational data uncertainty, and enable data and analytical products to feed directly into models. Data telemetry systems also are being updated to allow for two-way communications and more frequent transmission of data to the internet. 


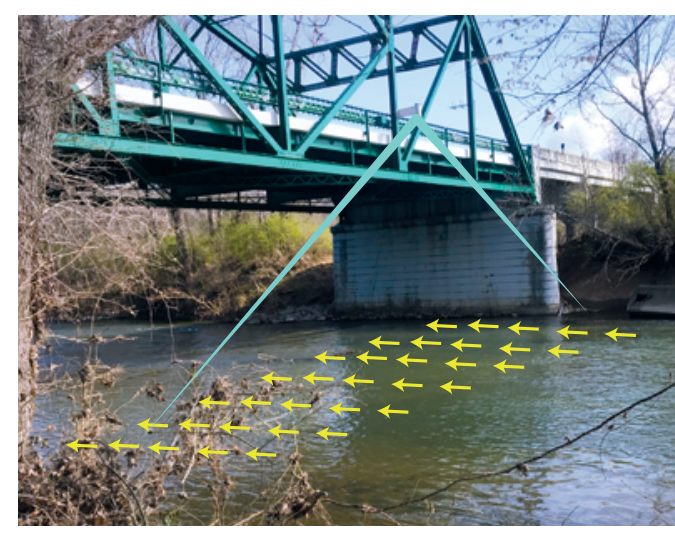

The USGS is advancing the use of large-scale particle-image velocimetry (LSPIV)—a method that uses innovative video analyzation techniquesto measure streamflow. Photograph by Mike Woodside, U.S. Geological Survey.

\section{Emerging and Innovative Technologies}

The NGWOS aims to foster innovation and development of monitoring technologies and methodologies to make data more affordable and more rapidly available. Monitoring innovations also are expected to lead to more types of data at higher temporal and spatial frequencies. Innovation testing sites will be identified on main-stem streams and small streams within NGWOS watersheds. These locations will provide a platform for rigorous, transparent, and reproducible testing of emerging and innovative monitoring technologies by the USGS and other entities. Technologies of interest include radar and image velocimetry for remotely sensing surface-water velocities, dronemounted ground-penetrating radar for measuring bathymetry for improving flow estimates, new sensors for monitoring continuous water-quality and suspended sediment, and others. The application and benefits of these innovations will extend beyond the NGWOS watersheds and be incorporated into routine operation of USGS monitoring networks.

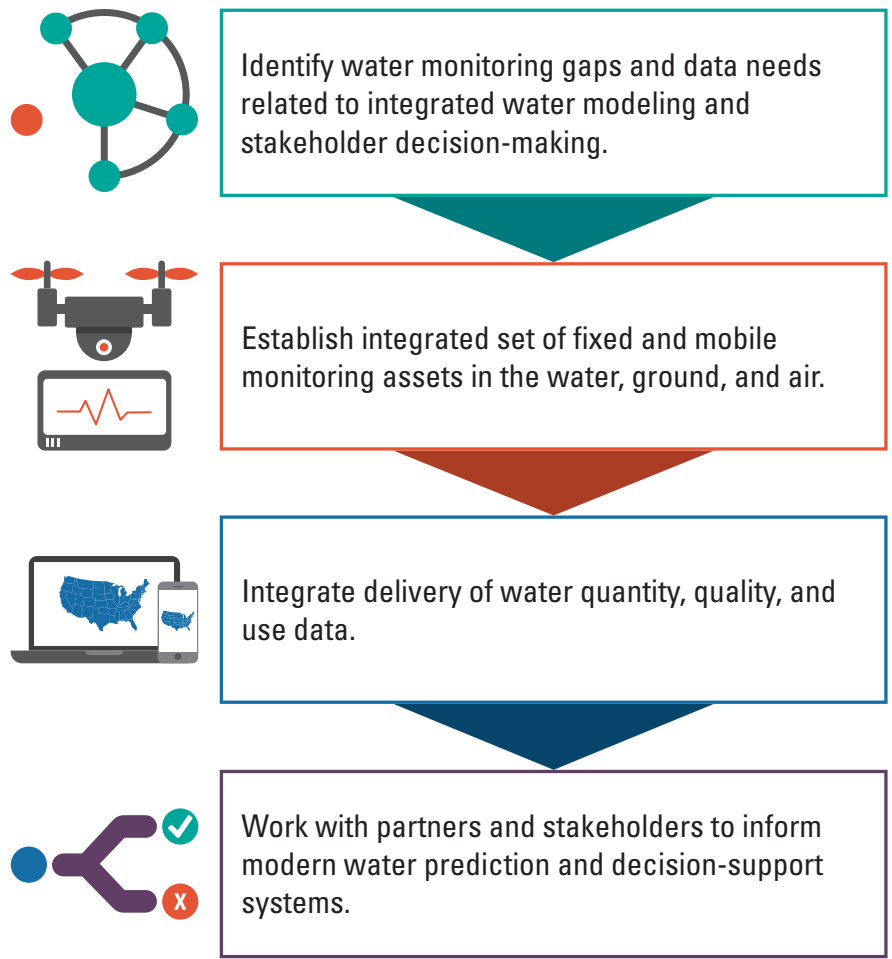

The USGS's NGWOS, when fully implemented, will provide real-time data on water quantity and quality. USGS partner and stakeholder needs are helping to inform the NGWOS design so that the data and information generated by the NGWOS will help them anticipate water shortages more accurately and react to water hazards more quickly.

\section{Delaware River Basin Pilot}

In 2018, the USGS began a pilot of the NGWOS in the Delaware River Basin. This pilot is providing an opportunity to demonstrate a water-observing system that will support innovative modern water prediction and decision-support systems in a nationally important, complex interstate river system. The following are some of the initial NGWOS activities in the Delaware River Basin:

- New streamgages addressing key monitoring gaps to better quantify the amount, temperature, and conductivity of water in headwater and tributary streams and to track the flux of salt water in the main stem of the Delaware River

- Webcams and drone-mounted sensors for operational and science applications

- Limited-scale soil moisture, snowpack, evapotranspiration, water-use, and groundwater/surface water interaction monitoring

- Enhanced two-way communication equipment at existing streamgages to enable remote operation and troubleshooting of monitoring equipment

- Cellular and satellite redundancy to ensure data are delivered during critical streamflow periods

- Innovation sites for testing a suite of new water-quality sensors and noncontact streamflow measurement technologies, and remote sensing of water quantity, quality, and use

\section{Reference Cited}

National Academies of Sciences, Engineering, and Medicine, 2018, Future water priorities for the Nation-Directions for the U.S. Geological Survey Water Mission Area: Washington, D.C., The National Academies Press, 96 p. [Also available at https://doi.org/10.17226/25134.]

\section{For more information:}

U.S. Geological Survey Water Resources Mission Area Groundwater and Streamflow Information Program https://www.usgs.gov/water-resources/groundwater-andstreamflow-information

Banner photograph by Jack Anstey, Unsplash, February 28, 2018.

By Sandra M. Eberts, Chad R. Wagner, and Michael D. Woodside 